Supplement of Biogeosciences, 13, 3991-4004, 2016

http://www.biogeosciences.net/13/3991/2016/

doi:10.5194/bg-13-3991-2016-supplement

(C) Author(s) 2016. CC Attribution 3.0 License.

(c) (1)

Supplement of

\title{
Analysing the uncertainty of estimating forest carbon stocks in China
}

Tian Xiang Yue et al.

Correspondence to: Tian Xiang Yue (yue@1reis.ac.cn) and Yi Fu Wang (wangyf@1reis.ac.cn)

The copyright of individual parts of the supplement might differ from the CC-BY 3.0 licence. 


\section{Supplement 1: HASM-SOA}

In terms of the fundamental theorem of surfaces, a surface is uniquely defined by the first and the second fundamental coefficients. The first fundamental coefficients are used to express the intrinsic geometric properties that do not depend on the shape of the surface, but only on measurements that we can carry out while on the surface itself. The second fundamental coefficients reflect the local warping of the surface, which can be observed from outside the surface (Yue et al. 2016).

If $\left\{\left(x_{i}, y_{j}\right)\right\}$ is an orthogonal division of a computational domain and $h$ represents the simulation step length, the central point of the lattice $\left(x_{\mathrm{i}}, y_{\mathrm{j}}\right)$ could be expressed as $(0.5 h+(i-1) h, 0.5 h+(j-1) h)$, in which $i=0,1,2, \ldots, I, I+1$ and $j=0,1,2, \ldots, J, J+1$. If $f_{\mathrm{i}, \mathrm{j}}^{(n)}(n \geq 0)$ represents the iterants of $f(x, y)$ at $\left(x_{\mathrm{i}}, y_{\mathrm{j}}\right)$ in the nth iterative step, in which $\left\{f_{i, j}^{(0)}\right\}$ are results from SOA, the iterative formulation of the master equation set of the method for high accuracy surface modeling (HASM) can be formulated as (Yue et al. 2013b; Zhao and Yue 2014 a, b),

$$
\begin{aligned}
& \frac{-f_{i+2, j}^{(n+1)}+16 f_{i+1, j}^{(n+1)}-30 f_{i, j}^{(n+1)}+16 f_{i-1, j}^{(n+1)}-f_{i-2, j}^{(n+1)}}{12 h^{2}} \\
& =\left(\Gamma_{11}^{1}\right)_{i, j}^{(n)} \frac{f_{i+1, j}^{(n)}-f_{i-1, j}^{(n)}}{2 h}+\left(\Gamma_{11}^{2}\right)_{i, j}^{(n)} \frac{f_{i, j+1}^{(n)}-f_{i, j-1}^{(n)}}{2 h}+\frac{L_{i j}^{(n)}}{\sqrt{E_{i, j}^{(n)}+G_{i, j}^{(n)}-1}} \\
& \frac{-f_{i, j+2}^{(n+1)}+16 f_{i, j+1}^{(n+1)}-30 f_{i, j}^{(n+1)}+16 f_{i, j-1}^{(n+1)}-f_{i, j-2}^{(n+1)}}{12 h^{2}} \\
& =\left(\Gamma_{22}^{1}\right)_{i, j}^{(n)} \frac{f_{i+1, j}^{(n)}-f_{i-1, j}^{(n)}}{2 h}+\left(\Gamma_{22}^{2}\right)_{i, j}^{(n)} \frac{f_{i, j+1}^{(n)}-f_{i, j-1}^{(n)}}{2 h}+\frac{N_{i j}^{(n)}}{\sqrt{E_{i, j}^{(n)}+G_{i, j}^{(n)}-1}} \\
& \frac{f_{i+1, j+1}^{(n+1)}-f_{i+1, j}^{(n+1)}-f_{i, j+1}^{(n+1)}+2 f_{i, j}^{(n+1)}+f_{i-1, j}^{(n+1)}-f_{i, j-1}^{(n+1)}+f_{i-1, j-1}^{(n+1)}}{2 h^{2}}
\end{aligned}
$$


$=\left(\Gamma_{12}^{1}\right)_{i, j}^{(n)} \frac{f_{i+1, j}^{(n)}-f_{i-1, j}^{(n)}}{2 h}+\left(\Gamma_{12}^{2}\right)_{i, j}^{(n)} \frac{f_{i, j+1}^{(n)}-f_{i, j-1}^{(n)}}{2 h}+\frac{M_{i j}^{(n)}}{\sqrt{E_{i, j}^{(n)}+G_{i, j}^{(n)}-1}}$

where $E_{\mathrm{i}, \mathrm{j}}^{(n)}, F_{\mathrm{i}, \mathrm{j}}^{(n)}$ and $G_{\mathrm{i}, \mathrm{j}}^{(n)}$ are the iterants of the first fundamental coefficients at the $n$th iterative step; $L_{\mathrm{i}, \mathrm{j}}^{(n)}, \boldsymbol{M}_{\mathrm{i}, \mathrm{j}}^{(\mathbf{n})}$ and $N_{\mathrm{i}, \mathrm{j}}^{(n)}$ represent the iterants of the second fundamental coefficients at the $n$th iterative step; $\left(\Gamma_{11}^{1}\right)_{i, j}^{(n)},\left(\Gamma_{11}^{2}\right)_{i, j}^{(n)},\left(\Gamma_{22}^{1}\right)_{i, j}^{(n)}$ and $\left(\Gamma_{22}^{2}\right)_{i, j}^{(n)}$ the iterants of the Christoffel symbols of the second kind at the $n$th iterative step, which depend only upon the first fundamental coefficients and their derivatives.

The matrix formulation of HASM master equations can be respectively expressed as,
$\mathbf{A} \cdot \mathbf{z}^{(n+1)}=\mathbf{d}^{(n)}$

$$
\begin{aligned}
& \mathbf{B} \cdot \mathbf{z}^{(n+1)}=\mathbf{q}^{(n)} \\
& \mathbf{C} \cdot \mathbf{z}^{(n+1)}=\mathbf{p}^{(n)}
\end{aligned}
$$

where $\mathbf{A}, \mathbf{B}$ and $\mathbf{C}$ represent coefficient matrices of the first equation, the second equation and the third equation; and $\mathbf{d}^{(n)}, \mathbf{q}^{(n)}$ and $\mathbf{p}^{(n)}$ are right-hand side vectors of the three equations respectively; $\mathbf{z}^{(n+1)}=\left(f_{1,1}^{(n+1)}, \ldots, f_{1, J}^{(n+1)}, \ldots \ldots, f_{I, 1}^{(n+1)}, \ldots, f_{I, J}^{(n+1)}\right)^{T}=\left(z_{1}^{(n+1)}, \ldots, z_{J}^{(n+1)}, \ldots, z_{(I-1) \cdot J+1}^{(n+1)}, \ldots, z_{I \cdot J}^{(n+1)}\right)^{T}$ $f_{i, j}^{(n)}$ is the value of the $n$th iteration of $f(x, y)$ at grid cell $\left(x_{i}, y_{i}\right)$; $z_{(i-1) \cdot J+j}^{(n+1)}=f_{i, j}^{(n+1)}$ for $1 \leq i \leq I, 1 \leq j \leq J$.

If $\bar{f}_{i, j}$ is BCS at the pth sample plot $\left(x_{i}, y_{j}\right), s_{p,(i-1) \times J+j}=1$, and $k_{p}=\bar{f}_{i, j}$. There is only one non-zero element, 1 , in every row of the coefficient matrix, $\mathbf{S}$, making it a sparse matrix. The solution procedure of HASM, taking the BCS at the sampled plots as its optimum control constraints and results from SOA as its driving 
field, can be transformed into solving the following linear equation set in terms of least squares principles,

$$
\left[\begin{array}{llll}
\mathbf{A}^{T} & \mathbf{B}^{T} & \mathbf{C}^{T} & \lambda \cdot \mathbf{S}^{T}
\end{array}\right]\left[\begin{array}{c}
\mathbf{A} \\
\mathbf{B} \\
\mathbf{C} \\
\lambda \cdot \mathbf{S}
\end{array}\right] \mathbf{z}^{(n+1)}=\left[\begin{array}{llll}
\mathbf{A}^{T} & \mathbf{B}^{T} & \mathbf{C}^{T} & \lambda \cdot \mathbf{S}^{T}
\end{array}\right]\left[\begin{array}{c}
\mathbf{d}^{(n)} \\
\mathbf{q}^{(n)} \\
\mathbf{p}^{(n)} \\
\lambda \cdot \mathbf{k}
\end{array}\right]
$$

The parameter $\lambda$ is the weight of the sample plots and determines the contribution of the sample plots to the simulated surface. $\lambda$ could be a real number, which means all sample plots have the same weight, or a sector, which means every sample plot has its own weight. An area affected by a sample plot in a heterogeneous region is smaller than in a homogeneous region. Therefore, a smaller value of $\lambda$ is selected in a heterogeneous region and a bigger value of $\lambda$ is selected in a homogeneous region. 